\section{Reimplante ureteral asistido por robot: técnica quirúrgica y revisión}

\author{
Corona-Montes VE, ${ }^{1}$ García-Nares SII, ${ }^{2}$ Zapata-González A
}

\section{RESUMEN}

La mayor parte de las veces las lesiones ureterales son una iatrogenia secundaria a procedimientos quirúrgicos, como la histerectomía por cualquiera de sus vías. La incidencia se incrementa en procedimientos quirúrgicos de mínima invasión y estenosis ureterales por diferentes padecimientos de la vía urinaria. El reimplante ureteral es un procedimiento de dominio obligado del urólogo. Su acceso, a partir del año 2003, es por vía robot asistida.

Uno de los objetivos de esta revisión, es describir la técnica quirúrgica del reimplante ureteral asistida por robot y revisar la bibliografía de este procedimiento.

Se detalla la técnica quirúrgica utilizada en la atención de una paciente de 46 años de edad con lesión ureteral secundaria a histerectomía.

Los resultados mostrados por los diferentes grupos que realizan reimplante ureteral robot asistido muestran un 90\% de lesiones ureterales distales la mayoría resueltas por la vía extra vesical.

Hoy día muchos grupos realizan cirugía ginecológica asistida con robot y el incidente ureteral es frecuente; por esto, una alternativa resolutiva es el uso del sistema quirúrgico Da Vinci, por la gran precisión en la resolución y la correcta reconstrucción ureteral que permiten la visión 3D y la movilidad y facilidad para la sutura.

PALABRAS CLAVE: reimplante ureteral, lesión ureteral, asistencia robótica.

Rev Mex Urol. 2017 March;77(2):158-165.

\section{Robotic-assisted ureteral reimplantation: Surgical technique and review}

\author{
Corona-Montes VE, ${ }^{1}$ García-Nares SII, ${ }^{2}$ Zapata-González A
}

\section{Abstract}

The majority of ureteral lesions are iatrogenic and are usually secondary to surgical procedures, such as hysterectomy performed with any technique. Injury is more common in minimally invasive surgery, as
${ }^{1}$ Departamento de Urología, Hospital General de México Dr. Eduardo Liceaga, Universidad Nacional Autónoma de México (UNAM), México.

${ }^{2}$ Centro de Cirugía Robótica, Hospital Ángeles del Pedregal, México.

Recibido: septiembre 2016

Aceptado: enero 2017

Correspondencia

Víctor Enrique Corona Montes

urocorona@hotmail.com

Este artículo debe citarse como

Corona-Montes VE, García-Nares Sigiberto II, Zapata-González A. Reimplante ureteral asistido por robot: técnica quirúrgica y revisión. Rev Mex Urol. 2017 mar;77(2):158-165. 
well as resulting from ureteral stricture due to different pathologies of the urinary tract. Ureteral reimplantation is an essential part of the urologist's armamentarium. The robotic-assisted approach has been carried out since 2003.

One of the objectives of this article was to describe the surgical technique of robotic-assisted ureteral reimplantation and to provide a review of the literature on this procedure.

Described herein are the details of the surgical technique performed on a 46-year-old patient with a ureteral lesion secondary to hysterectomy.

The results from different groups that have performed robotic-assisted ureteral reimplantation show that $90 \%$ of distal ureteral injuries are resolved through the extravesical technique.

Many groups currently perform robotic-assisted gynecologic surgery and the incidence of ureteral lesions is frequent. The da Vinci Surgical System is an alternative that provides great precision in injury resolution, with correct ureteral reconstruction through three-dimensional vision, improved mobility, and ease in suturing.

KEY WORDS: ureteral reimplantation, ureteral injury, robotic-assisted

\author{
${ }^{1}$ Departamento de Urología, Hospital \\ General de México Dr. Eduardo Liceaga, \\ Universidad Nacional Autónoma de México \\ (UNAM), México. \\ ${ }^{2}$ Centro de Cirugía Robótica, Hospital Án- \\ geles del Pedregal, México. \\ Correspondence \\ Víctor Enrique Corona Montes \\ urocorona@hotmail.com
}

\section{ANTECEDENTES}

La lesión ureteral en el adulto puede requerir el reimplante de éste como tratamiento primario. En muchos casos es frecuente la excisión del sitio de lesión y su anastomosis a la vejiga, dependiendo de la longitud y altura de la lesión. Algunas alternativas son: flap de Boari, psoas hitch o el reimplante ureteral directo. Aparentemente, la cirugía de mínima invasión, laparoscópica o robótica, podría ponerse en duda; sin embargo, es posible realizar estas reconstrucciones ureterales por vía laparoscópica o robótica, por supuesto con ventajas claras de la robótica para la manipulación del tejido fino. El reimplante ureteral con vejiga psoica fue descrito por primera vez por Zimmerman y colaboradores en $1960 .{ }^{1}$ Reddy y Evans describieron la primera ureteroneocistostomía por vía laparoscópica en adultos. ${ }^{2}$ Olsen y colaboradores fueron los primeros en utilizar el sistema Da Vinci para efectuar un reimplante ureteral robótico, con acceso intravesical, en un modelo porcino. ${ }^{3}$

El primer reimplante ureteral laparoscópico asistido por robot se efectuó en 2003 por Yohannes, quien lo realizó por estenosis ureteral; ${ }^{4}$ desde entonces, este acceso ha ganado adeptos, y Uberoi, en 2007, reportó una ureteroneocistostomía indicada por tumor ureteral. $^{5}$

Muchas de las ventajas del robot son inherentes a todos los procedimientos realizados mediante la técnica laparoscópica; el cirujano es capaz de trabajar en la pelvis profunda con adecuada visión y magnificación en tercera dimensión, la sutura intracorporal puede efectuarse en un campo quirúrgico favorable y el neumoperitoneo 
ofrece ventaja hemostática para mejorar la visión y limitar el sangrado. Además el robot representa, de forma más cercana, la técnica convencional respecto de la técnica laparoscópica pura. ${ }^{6}$ Por lo general, la posición del paciente es en litotomía con Trendelenburg, para colocar al robot entre las piernas del paciente, lo que limita el acceso retrógrado a la vejiga para derivación ureteral, por lo que se ha propuesto el acoplamiento lateral del robot. $^{7}$

Se describe la técnica quirúrgica de ureteroneocistostomía asistida por robot en patología distal ureteral y se revisa la bibliografía relacionada con los resultados. En relación con el reimplante ureteral asistido por robot se describen los pasos de la técnica quirúrgica en una lesión ureteral izquierda, secundaria a histerectomía por miomatosis uterina.

\section{MATERIALES Y MÉTODOS}

Estudio descriptivo y retrospectivo efectuado con base en la revisión de la bibliografía del reimplante ureteral asistido por robot.

\section{Descripción de la técnica quirúrgica}

Se coloca al paciente en decúbito dorsal, con las piernas en discreta abducción y Trendelenburg a $45^{\circ}$. Se coloca una sonda Foley de 18 french. El robot se coloca a los pies del paciente, en medio de las piernas. La intervención se lleva a cabo con tres brazos de robot. La posición del trócar (12 mm) para la cámara es de $2 \mathrm{~cm}$ arriba de la cicatriz umbilical, en la línea media; 2 trócares de $8 \mathrm{~mm}$ robóticos cada uno a lo largo de la línea medio clavicular, un centímetro abajo de la altura umbilical. Un puerto de $5 \mathrm{~mm}$ en la línea paraaxilar lateral a la cresta iliaca, a $4 \mathrm{~cm}$ de la misma y un puerto de $10 \mathrm{~mm}$ que forma un triángulo entre el puerto de la cámara y el puerto robótico medio clavicular, ambos para el asistente. No se utilizó el cuarto brazo del robot para que ello no repercutiera en el costo operativo. Figura 1

Después de instalar el sistema quirúrgico Da Vinci se procede a identificar el sitio de lesión
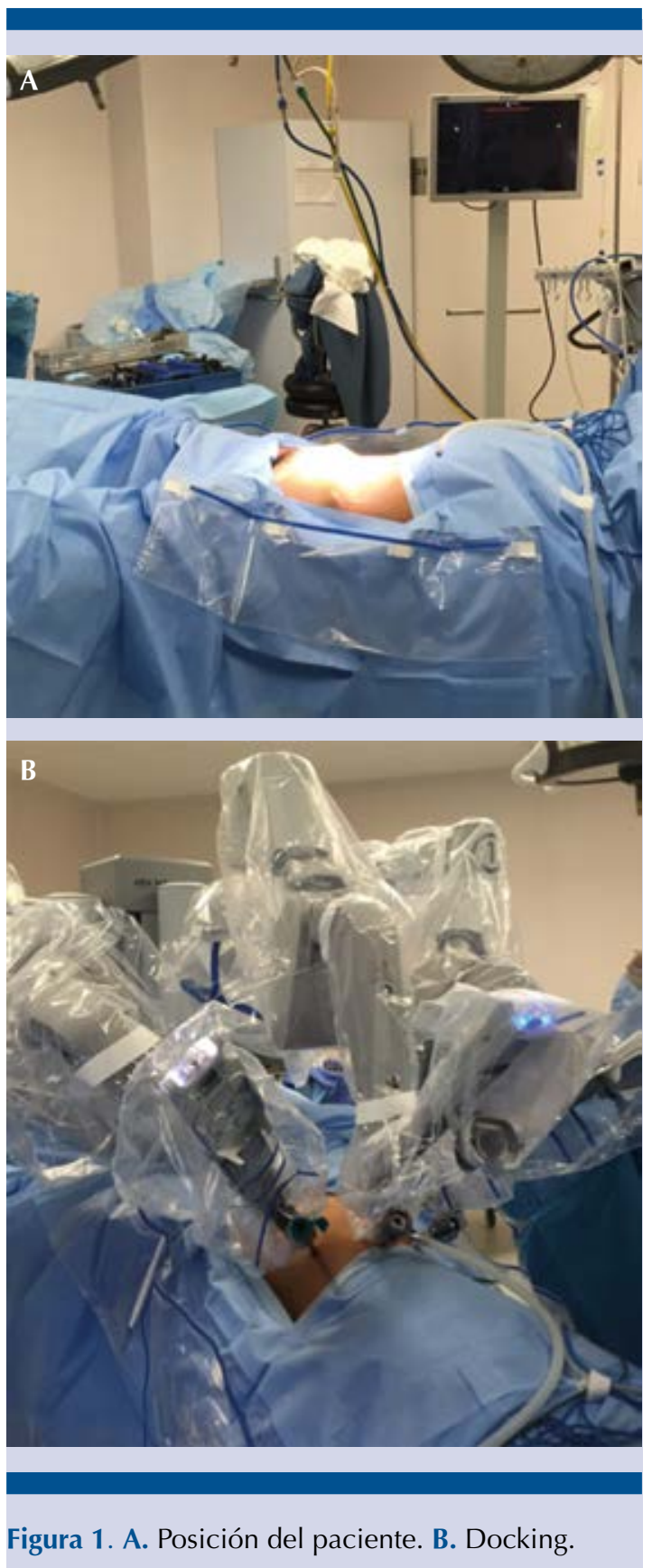
ureteral distal izquierda con lo que se logra la movilización ureteral sin afectar a la mayor cantidad de vasos periureterales y conseguir mejor aporte sanguíneo. Enseguida se procede a la resección del tejido fibroso periureteral de la lesión ureteral para calcular la longitud hacia la vejiga y determinar el tipo de técnica a utilizar, para el caso de un psoas hitch asistido con robot. La vejiga se moviliza a partir del ligamento umbilical izquierdo para liberar la tensión; para una mejor exposición y unión del reimplante ureteral, el espacio de Retzius se baja, aproximadamente, $3 \mathrm{~cm}$. Se procede a realizar el punto al psoas hitch para dar estabilidad vesical. Se selecciona el espacio para la incisión vesical y futuro sitio del reimplante y el espacio para el túnel submucoso. Figura 2

Se espatuló el uréter y se colocó una sutura de fijación para iniciar su anastomosis y su aproximación al sitio de apertura vesical, visualizando la mucosa. Enseguida, se continúa con la sutura de la unión uretero-vejiga, previa colocación del catéter ureteral doble J y se completa mucosamucosa dejando el catéter ureteral doble J en el interior. A continuación se cierra el paso del túnel submucoso y se verifica que la unión uretero-vejiga esté libre de tensión. Se verifica que el cierre haya quedado completamente hermético. En caso de sospecha de devascularización importante se requerirá un parche de epiplón. Figura 3

\section{DISCUSIÓN}

El reimplante ureteral es una habilidad decisiva que el urólogo debe dominar porque puede utilizarse para reparar lesiones iatrogénicas, reflujo vésico-ureteral, estenosis ureterales benignas o tumores del uréter. ${ }^{8}$ La ureteroneocistostomía es el patrón de referencia de la reparación ureteral distal que no es posible reparar por vía endoscópica. ${ }^{9}$

El reimplante intravesical ha sido un procedimiento exitoso, inclusive con mayor éxito que el reimplante extravesical; sin embargo, la incisión vesical se asocia con comorbilidades como hematuria y cistitis. ${ }^{10}$ Durante los últimos años, como alternativa a la cirugía por reflujo vésicoureteral se introdujo la inyección subureteral de material sintético, que no ofrece resultados equiparables a los obtenidos con el reimplante abierto. ${ }^{11}$ Hace poco, el reimplante ureteral laparoscópico demostró que es una técnica útil, ${ }^{10}$ con tasas de éxito de $79-100 \%$ intra o extravesical $^{12-13}$ en el tratamiento de padecimientos ureterales benignos y malignos. Sin embargo,

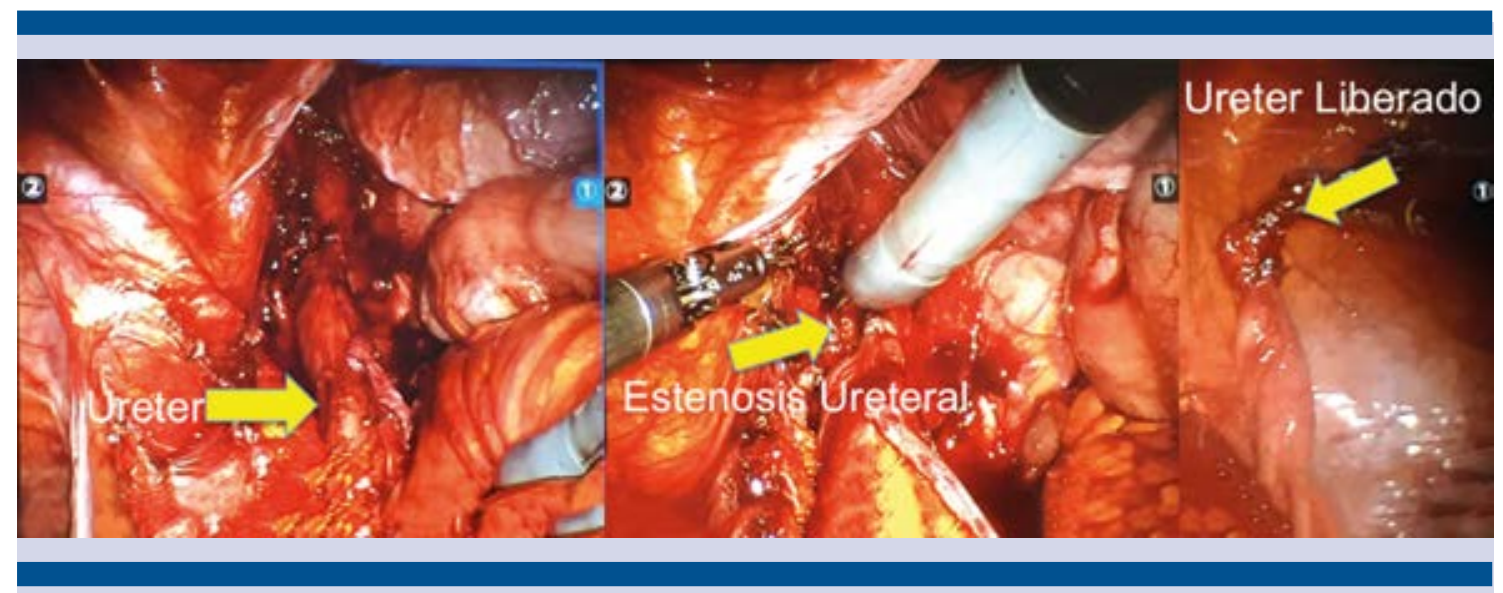

Figura 2. Disección ureteral. 


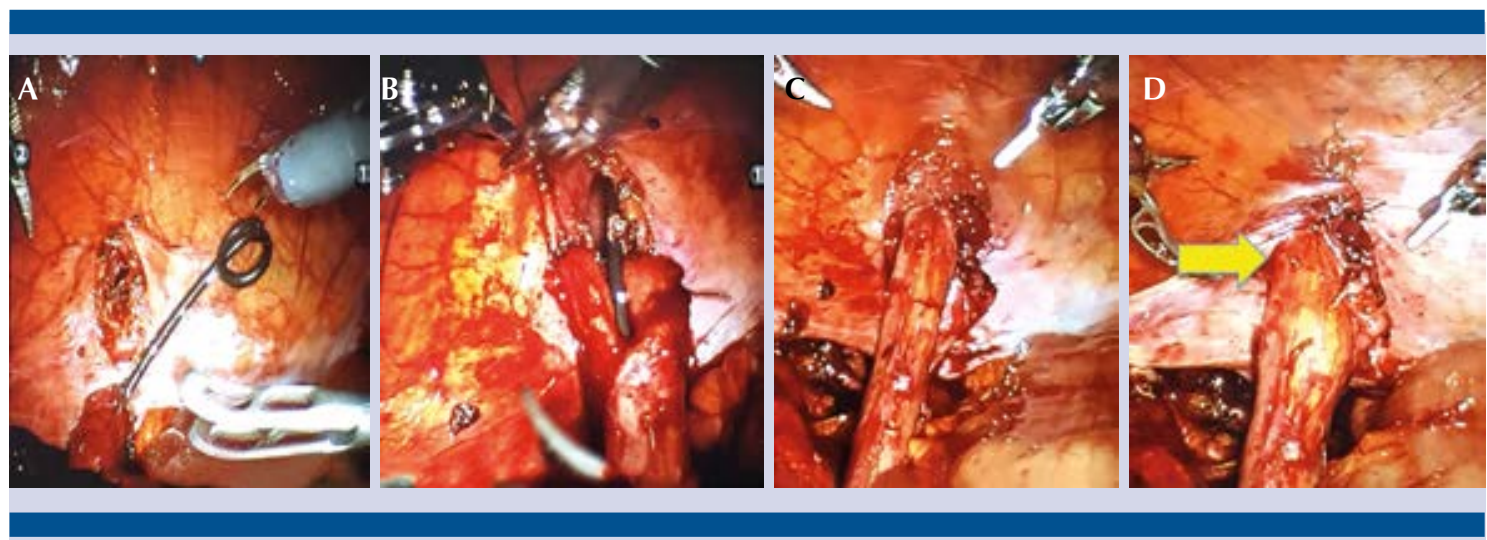

Figura 3. A. Resección B. Colocación doble J. C. Anastomosis. D. Antirreflujo.

se trata de una técnica demandante y de largo tiempo quirúrgico debido a la manipulación de instrumentos laparoscópicos y tejidos bajo visión en 2D; en cambio, el reimplante ureteral asistido por robot es una alternativa en aumento en todo el mundo desde hace más de una década. ${ }^{10}$

La revisión se homogeneizó con autores descritos con características similares en sus series de casos, de los que se obtuvo una media de edad de 50.2 años (41-63). En todos los casos el procedimiento efectuado fue la uretero-neocistostomía asistida por robot, casos secundarios a lesiones del uréter distal, a excepción de Shuan y su grupo, quienes reportan lesiones medio-distales. (Cuadro 1) Los tiempos de cirugía tuvieron una media de 241 min (182 - 282), el sangrado transoperatorio fue de $74 \mathrm{~mL}(0-123)$, los días de estancia hospitalaria correspodienron a 5 días (2.5-12) con una recurrencia, en siete casos, de 74 para el grupo homogéneo. Cuadro 2

En 2005, Peters y Woo reportaron su experiencia inicial en el reimplante ureteral laparoscópico asistido por robot con técnica intravesical, con éxito de $92 \%$, aunque un paciente tuvo fuga de orina debia a mal cierre de uno de los puertos de trabajo. ${ }^{14} \mathrm{El}$ reimplante ureteral extravesical robótico se lleva a cabo con más frecuencia que el intravesical, con resultados similares a
Cuadro 1. Descriptiva del procedimiento

\begin{tabular}{|c|c|c|c|}
\hline Autor & Edad & Procedimiento & Localización \\
\hline $\begin{array}{l}\text { Lee y su } \\
\text { grupo }\end{array}$ & 52 & R UNC & Distal \\
\hline $\begin{array}{l}\text { Shuan y } \\
\text { colaboradores }\end{array}$ & 46 & R UNC & Medio Distal \\
\hline $\begin{array}{l}\text { Patil y } \\
\text { coautores }\end{array}$ & 41 & R UNC & Distal \\
\hline $\begin{array}{l}\text { Schimpf y } \\
\text { su grupo }\end{array}$ & 63 & R UNC & Distal \\
\hline $\begin{array}{l}\text { Isac y } \\
\text { colaboradores }\end{array}$ & 49 & R UNC & Distal \\
\hline \multirow[t]{2}{*}{$\begin{array}{l}\text { Musch y } \\
\text { colaboradores }\end{array}$} & 59 & R UNC & Distal \\
\hline & 50.2 & & \\
\hline
\end{tabular}

la laparoscopia pura. Además, se conserva una anatomía adecuada para el acceso ureteral retrógrado, con menores: hematuria, espasmo vesical, estancia hospitalaria y cateterismo ureteral, en comparación con la técnica intravesical. ${ }^{8}$

Smith y colaboradores compararon, en 2011, el tiempo quirúrgico del reimplante extravesical robótico y el reimplante ureteral abierto, con técnica de Cohen, en pacientes pediátricos; fue mayor con robot, pero la estancia hospitalaria y el requerimiento de analgésicos fueron menores. ${ }^{15}$ La serie más grande de reimplante ureteral robótico comparado con reimplante ureteral 
Cuadro 2. Variables Perioperatorias

\begin{tabular}{|c|c|c|c|c|c|}
\hline Autor & $\begin{array}{c}\mathbf{T} \text { de } \mathbf{Q x} . \\
\text { (min) }\end{array}$ & $\begin{array}{c}\text { Sangrado } \\
(\mathrm{mL})\end{array}$ & $\begin{array}{c}\text { Estancia } \\
\text { (días) }\end{array}$ & Recurrencia & $\begin{array}{c}\text { Casos } \\
(\#)\end{array}$ \\
\hline $\begin{array}{l}\text { Lee } \\
\text { et al. }\end{array}$ & 211 & 102 & 2.8 & 2 & 10 \\
\hline $\begin{array}{l}\text { Shuan } \\
\text { et al. }\end{array}$ & 282 & 123 & 2.5 & 1 & 13 \\
\hline $\begin{array}{l}\text { Patil } \\
\text { et al. }\end{array}$ & 208 & 48 & 4.3 & 0 & 12 \\
\hline $\begin{array}{l}\text { Schimpf } \\
\text { et al. }\end{array}$ & 182 & 10 & 2.0 & 2 & 5 \\
\hline $\begin{array}{l}\text { Isac } \\
\text { et al. }\end{array}$ & 279 & 100 & 3 & 2 & 25 \\
\hline $\begin{array}{l}\text { Musch } \\
\text { et al. }\end{array}$ & 271 & 0 & 12 & 0 & 9 \\
\hline Medias & 241 & 74 & 5 & 7 & 74 \\
\hline
\end{tabular}

T de Qx: tiempo quirúrgico.

abierto es la de Isac (2013) quien comparó 25 reimplantes robóticos versus 41 reimplantes convencionales. Se demostró la ventaja del robot en relación con la estancia hospitalaria, sangrado y necesidad de analgésicos, mientras que la técnica convencional demostró menor tiempo quirúrgico, aunque sin diferencia significativa en el riesgo de reoperación. ${ }^{16}$

Existen pocos estudios, incluso en población pediátrica, de reimplante ureteral por reflujo vésico-ureteral, aunque con resultados prometedores, ${ }^{9}$ con $3 \%$ de la causalidad. La primera razón fueron las estenosis ureterales con $41 \%$ y la histerectomía con $37 \%$. Figura 4

Al afinar detalles acerca de los aspectos técnicos de esta operación, se incluye la anastomosis libre de tensión e impermeable al agua, con aproximación de mucosa de tejido viable; además del reimplante, el urólogo debeser capaz de formar una vejiga psoica o colgajo de Boari en caso necesario, para cumplir con los requisitos reconstructivos. La sutura y manipulación de tejidos en el limitado espacio de la pelvis se efectúa con mayor facilidad con el robot que con la laparoscopia convencional. ${ }^{12}$ Este último

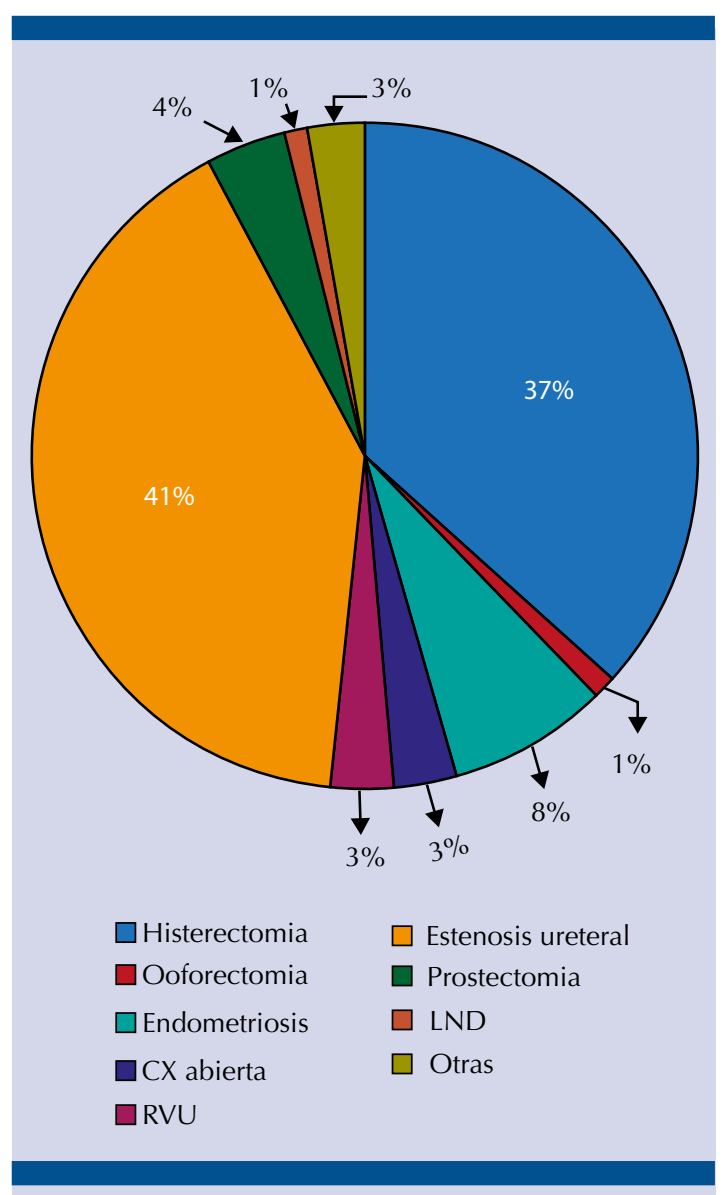

Figura 4. Razones del reimplante ureteral.

aspecto ha permitido la ejecución de técnicas mínimamente invasivas para tratar pacientes con carcinoma urotelial de la vía urinaria superior, con adecuado control oncológico y sin aumento de riesgo de la siembra tumoral. ${ }^{9}$

Durante los últimos años se han reportado resultados similares entre el reimplante abierto y robótico. Existen pocos estudios comparativos de la efectividad del reimplante ureteral abierto versus el robótico. ${ }^{12}$ La mayor parte de las series de reimplantes robóticos refieren tasas de éxito cercanas al $100 \%$; las complicaciones varían en las distintas series de 0 a $55 \% .{ }^{17-18}$ Figura 5 


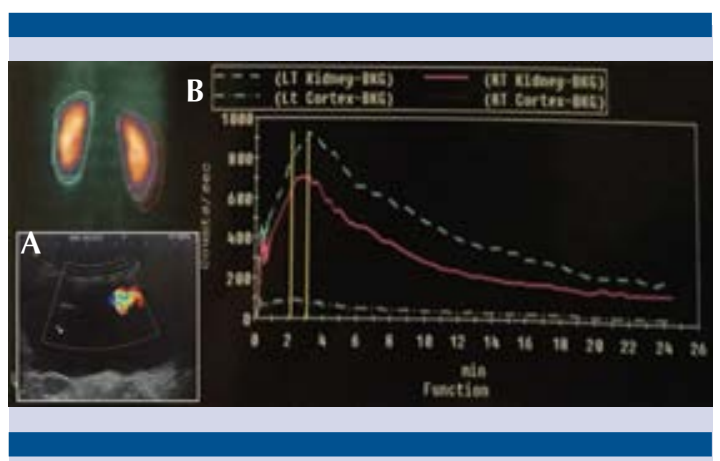

Figura 5. A. Jet ureteral en ultrasonido de control. B. MAG 3 .

\section{CONCLUSIÓN}

La información internacional de la cirugía reconstructiva del uréter distal asistida por robot es limitada; sin embargo, la reunida hasta la fecha es una técnica factible de fácil reproducción, sin sacrificar los principios de la cirugía abierta, con adecuada evolución funcional a corto plazo y excepcionales complicaciones posquirúrgicas graves. Sin duda, el tiempo quirúrgico disminuirá conforme más experiencia se adquiera con los reimplantes robóticos. Se espera que en el futuro el robot demuestre su superioridad en relación con el reimplante abierto, así como la plastia de la unión ureteropiélica laparoscópica asistida por robot superó al abordaje abierto; hoy día, las técnicas mínimamente invasivas son el patrón de referencia.

\section{FINANCIAMIENTO}

Los autores no recibieron ningún patrocinio para llevar a cabo este artículo.

\section{Conflicto de intereses}

Los autores declaran no tener conflicto de intereses.

\section{REFERENCIAS}

1. Zimmerman IJ, Precourt WE, Thompson CC. Direct ureterocysto-neostomy with the short ureter in the cure of ureterovaginal fistula. J Urol 1960; 83:113-115.

2. Reddy PK, Evans RM. Laparoscopic ureteroneocystostomy. J Urol 1994; 152:2057-2059.

3. Olsen LH, Deding D, Yeung CK et al. Computer assisted laparoscopic pneumovesical ureter reimplantation a.m. Cohen: initial experience in a pig model. APMIS 2003; 109: 23-25.

4. Yohannes P, Chiou RK, Pelinkovic D. Rapid communication: pure robot-assisted laparoscopic ureteral reimplantation for ureteral stricture disease: case report. J Endourol 2003; 17: 891-893.

5. Uberoi J, Harnisch B, Sethi AS, et al. Robot-assisted laparoscopic distal ureterectomy and ureteral reimplantation with psoas hitch. J Endourol 2007; 21:368-373; discussion 372-363.

6. Musch M, Hohenhorst L, Pailliart A, et al. Robot-assisted reconstructive surgery of the distal ureter: single institution experience in 16 patients. BJU Int 2013; 111: 773-783.

7. Slater R, Farber NJ, Riley JM, et al. Contemporary Series of Robotic-Assisted Distal Ureteral Reconstruction Utilizing Side Docking Position. Int Braz J Urol 2015; 41: 1154-1159.

8. Gorbonos A, McClung C. Ureteral Reimplantation in Adults: Open Versus Robotic. J Urol 2014; 192: 1023-1025.

9. Wason S, Lance R, Given R, et al. Robotic-Assisted Ureteral Re-implantation: A Case Series. J Laparoendosc Adv Surg Tech 2015; 25 (6): 503-507.

10. Hayashi Y, Mizuno K, Kurokawa S, et al. Extravesical robot-assisted laparoscopic ureteral reimplantation for vesicoureteral reflux: Initial experience in Japan with the ureteral advancement technique. Int J Urol 2014; 21, 1016-1021.

11. Elder JS, Diaz M, Caldamone AA et al. Endoscopic therapy for vesicoureteral reflux: a meta-analysis. I. Reflux resolution and urinary tract infection. J Urol 2006; 175: 716-722.

12. Lakshmanan Y, Fung LC. Laparoscopic extravesicular ureteral reimplantation for vesicoureteral reflux: recent technical advances. J Endourol 2000; 14: 589-593.

13. Riquelme $\mathrm{M}$, Aranda A, Rodriguez C. Laparoscopic extravesical transperitoneal approach for vesicoureteral reflux. $J$ Laparoendosc Adv Surg Tech 2006; 16: 312-316.

14. Peters CA, Woo R. Intravesical robotically assisted bilateral ureteral reimplantation. J Endourol 2005; 19: 618-621.

15. Smith RP, Oliver JL, Peters CA. Pediatric robotic extravesical ureteral reimplantation: comparison with open surgery. $J$ Urol 2011; 185: 1876-1881.

16. Isac W, Kaouk J, Altunrende F, et al. Robot-assisted ureteroneocystostomy: Technique and comparative outcomes. J Endourol 2013; 27: 318-323. 
17. Patil NN, Mottrie A, Sundaram B, Patel VR. Robotic-assisted laparoscopic ureteral reimplantation with psoas hitch: A multi-institutional, multinational evaluation. Urology 2008; 72: 47-50.
18. Schimpf MO, Wagner JR. Robot-assisted laparoscopic distal ureteral surgery. JSLS 2009; 13: 44-49.

19. Ziho L, Shailen S, Elton LI, et al Single-Surgeon Experience with Robot-Assisted Ureteroneocystostomy for Distal Ureteral Pathologies in Adults. Korean J Urol 2013;54:516-521.

\section{AVISO PARA LOS AUTORES}

Revista Mexicana de Urología tiene una nueva plataforma de gestión para envío de artículos. En: https://www.revisionporpares.com/index.php/RMUrol podrá inscribirse en nuestra base de datos administrada por el sistema Open Journal System (OJS) que ofrece las siguientes ventajas para los autores:

- Subir sus artículos directamente al sistema.

- Conocer, en cualquier momento, el estado de los artículos enviados, es decir, si ya fueron asignados a un revisor, aceptados con o sin cambios, o rechazados.

- Participar en el proceso editorial corrigiendo y modificando sus artículos hasta su aceptación final. 